\title{
Comparison of Maximal Gingival Display during Different Facial Expressions and Evaluation of Golden Percentage in a Section of North Indian Population: An AutoCAD Analysis
}

\author{
${ }^{1}$ Mahak Qadri, ${ }^{2}$ Pankaj Dhawan, ${ }^{3}$ Pankaj Madhukar, ${ }^{4}$ Piyush Tandan
}

\section{ABSTRACT}

Aim: To compare the amount of maximal gingival display among four different facial expressions and evaluate the golden percentage in a section of North Indian population.

Materials and methods: Photographs of a total of 100 subjects of North Indian origin with age ranging from 18 to 25 years will be taken and then assessed using AutoCAD ${ }^{\odot}$ software.

Results: Neither smile type (requested or Duchenne) revealed a significantly greater amount of gingival display above the maxillary central or lateral incisors, but the grimace of disgust and funnel-shaped expressions did. At the central and lateral incisors, the grimace of disgust and funnel-shaped expressions produced the greatest amounts of gingival display. At the maxillary canines and the first premolars, both smiles (requested and Duchenne) exhibited the largest amount of gingival display of the four facial expressions.

Conclusion: There exists a difference in gingival display among four different facial expressions over each tooth visible, and the golden percentage was found to be present in more than $99 \%$ of the subjects studied.

Clinical significance: This article considers gingival display as an important factor in rehabilitating esthetic smiles, and proposes the concept of the golden percentage as a more useful application in diagnosing and developing symmetry, dominance, and proportion for esthetically pleasing smiles.

Keywords: Esthetics, Facial expressions, Gingival display, Golden percentage, Smile.

How to cite this article: Qadri M, Dhawan P, Madhukar P, Tandan P. Comparison of Maximal Gingival Display during Different Facial Expressions and Evaluation of Golden Percentage in a Section of North Indian Population: An AutoCAD Analysis. Int J Prosthodont Restor Dent 2016;6(4):79-84.

Source of support: Nil

Conflict of interest: None

${ }^{1}$ Postgraduate Student, ${ }^{2}$ Head, ${ }^{3}$ Professor, ${ }^{4}$ Associate Professor

${ }^{1-4}$ Department of Prosthodontics and Crown and Bridge and Implants, Manav Rachna Dental College, Faridabad, Haryana India

Corresponding Author: Mahak Qadri, Postgraduate Student Department of Prosthodontics and Crown and Bridge and Implants, Manav Rachna Dental College, Faridabad, Haryana India, Phone: +8130513301, e-mail: mahak.qadri@yahoo.com

\section{INTRODUCTION}

Smiling is one of the simplest, most easily recognized, and yet confusing of all the possible facial expressions. Clearly, a significant influence on beauty lies in the lower one-third of the face. ${ }^{1}$ For an attractive smile, the position, form, and color of the upper central incisors are the most essential factors. Esthetic conditions related to gingival health and appearance are essential components of effective smile design. Inflamed, uneven gingival lines diminish the value from a pleasing smile. Blunt papilla and asymmetric gingival crests become part of an overall esthetic picture. ${ }^{2}$ Some amount of gingival display in the smile is certainly acceptable and, in many cases, is even esthetic and youthful appearing. A complete lack of gingival display is not as attractive. Excessive amount of gingival display - so called gummy smile - can render a smile as severe and unpleasant. ${ }^{3}$ The ancient Greeks believed that there was a constant proportion between large and small in the beauty of nature. Application of the golden proportion to dental esthetics was first documented by Levin ${ }^{4}$ in 1978 . He explained the association of proportion with esthetically pleasing smile and dentition. Snow ${ }^{5}$ advocated the use of the "golden percentage" as a means of applying the golden proportion across the midline to encompass the total canine-to-canine width (six anterior teeth). According to him, the proportional width of each tooth should be: Canine 10\%, lateral incisor $15 \%$, central incisor $25 \%$, central incisor $25 \%$, lateral incisor $15 \%$, and canine $10 \%$ of the total distance across the anterior segment. The principle of golden percentage in evaluation and treatment planning is of significant benefit in esthetic smile design. In the clinical application of smile design, the final relative width proportion of anterior teeth is ultimately dependent upon the arch form. In comparing the two arches that have teeth with identical mesiodistal widths, the wider arch form would have relatively narrower central incisors and relatively wider canines, less closely imitating the golden percentage relation. The narrower arch would have relatively wider central incisors and relatively narrower canines, more closely approximating the golden percentage. ${ }^{5}$ 
AIM

To compare the amount of maximal gingival display among four different facial expressions and evaluate the golden percentage in a section of North Indian population.

\section{MATERIALS AND METHODS}

The study was conducted in the Department of Prosthodontics and Crown and Bridge and Implants, Manav Rachna Dental College, Faridabad, India. In the present study, a total of 100 subjects of North Indian origin with age ranging from 18 to 25 years were randomly selected. The subjects thus selected for this study were young adults belonging to different states and of different age groups. To participate in this research project, individuals had to meet the following inclusion criteria: Dentulous subjects ranging from 18 to 25 years of age; well-aligned natural maxillary anterior teeth; completely dentate arch with no interdental spacing, no anterior restoration, and no history of orthodontic treatment. A participant informed consent form (PICF) was provided in both English and Hindi languages. The PICF was signed and explained to each participant before starting the procedure. Each subject was made to sit upright on a dental chair with the occlusal plane of the maxillary teeth parallel to the floor, and a standardized protocol for patient positioning and the focal distance (portrait $-100 \mathrm{~cm}$, teeth $-30 \mathrm{~cm}$ ) between the camera and the subjects' smile was maintained. The action units (AUs) developed by Ekman et $\mathrm{al}^{6}$ were used to explain to the participant what was required to accurately achieve the facial expressions. The first facial expression, a requested smile, was obtained simply by asking participants to "give me your biggest smile", which is not coded with AUs. The followings three facial expressions were coded with AUs: Duchenne smile (AUs 6, 12, 25), grimace of disgust (AUs 9, 10, 25), and funnel-shaped expression (AUs 9, 22, 25). The participants were also shown individual $8 \times 10$ inch color photographs of an unfamiliar person making an intense grimace of disgust and a funnel-shaped expression (Table 1).

A fixed focus of 1:10 was used for the full face and 1:1 for smile of each subject, with the EF $100 \mathrm{~mm} f / 2.8$ macro ultrasonic motor lens. ${ }^{7,8}$ The height of the Canon EOS 1200D DSLR camera mounted on a tripod (Traveller Mini Pro Tripod For Canon EOS 1200D) was adjusted individually according to the position of the subject's face and the teeth. ${ }^{9}$ Nine standardized photographs were taken for each subject: Portrait (closed lips), portrait (four facial expressions), maxillary anterior teeth (Duchenne Smile), and four facial expressions (closeup). In between each expression, participant was asked to rest the lips.

The images of the participants were then transferred to a computer (HP Pavilion i3 Notebook PC, Windows 7)
Table 1: Action units

\begin{tabular}{cl}
\hline Action unit & Definitions \\
\hline 6 & Muscles around eyes are contracted \\
& Infraorbital triangle is raised and cheeks are lifted \\
& superiorly \\
& Wrinkles are formed laterally from corners of eyes \\
& Skin along nose is drawn superiorly, which results in \\
& wrinkles to form lateral to and across bridge of nose \\
& Infraorbital triangle is pulled superiorly and results in \\
& infraorbital furrow to wrinkle and raise skin at lower \\
& eyelids \\
& Center of upper lip is pulled upward and separates \\
& lips \\
& Nostrils widen and rise \\
& Nasolabial furrow deepens \\
& Center of upper lip is pulled superiorly, while outer \\
& portions of lips are drawn up, but not as high \\
& Angular bend in shape of upper lip \\
& Infraorbital triangle is pushed superiorly \\
& Nasolabial furrow deepens as upper part of furrow \\
& rises \\
& Nostrils widen and rise \\
& Lips separate when action is strong \\
& Corners of lips are pulled laterally and superiorly \\
& Nasolabial furrow deepens, as it is pulled laterally \\
& and superiorly \\
& Lips are pushed outward to make funnel shape \\
& Lip corners are pulled in medially \\
& Lips are separated hard and soft tissues are exposed \\
&
\end{tabular}

in JPEG format and then one by one, the image was transferred and opened in the AutoCAD ${ }^{\odot}$ (Autodesk Inc. 2013, California, USA) analysis software (AutoCAD 2013 Version 19.0) (Figs 1 to 4).

The mathematical assessment of the photographs was done according to the methodology proposed by Walter et $\mathrm{al}^{10}$ and Snow. ${ }^{5}$ If a tooth's gingival zenith could not be identified, then that specific tooth was recorded as $0.00 \mathrm{~mm}$ for the given facial expression.

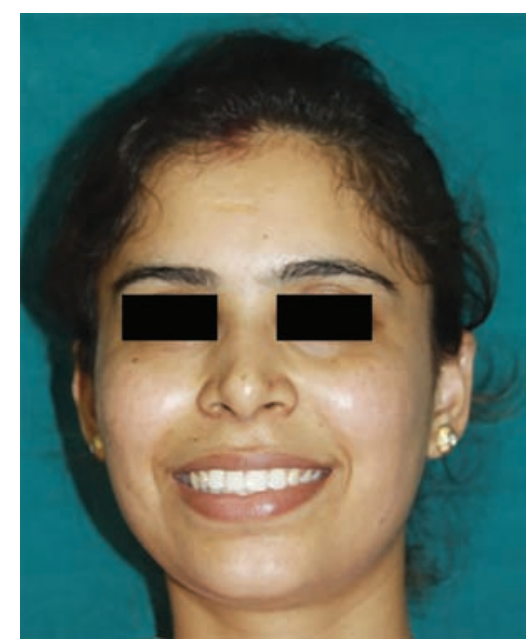

Fig. 1: Example of what subject may do when asked "give me your biggest smile" 


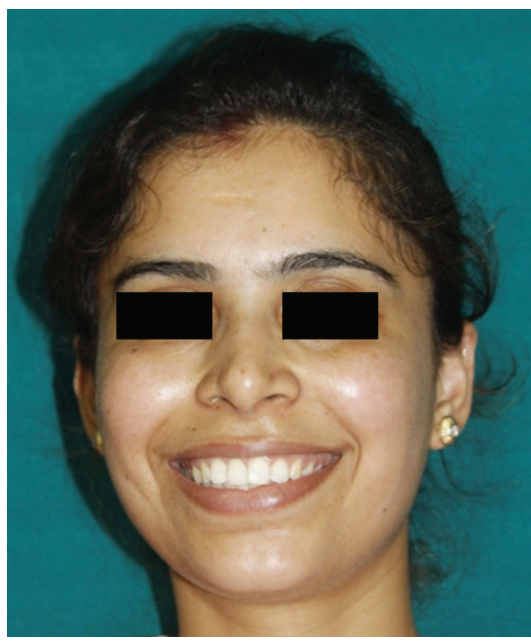

Fig. 2: Example of Duchenne smile (AUs 6, 12, and 25)

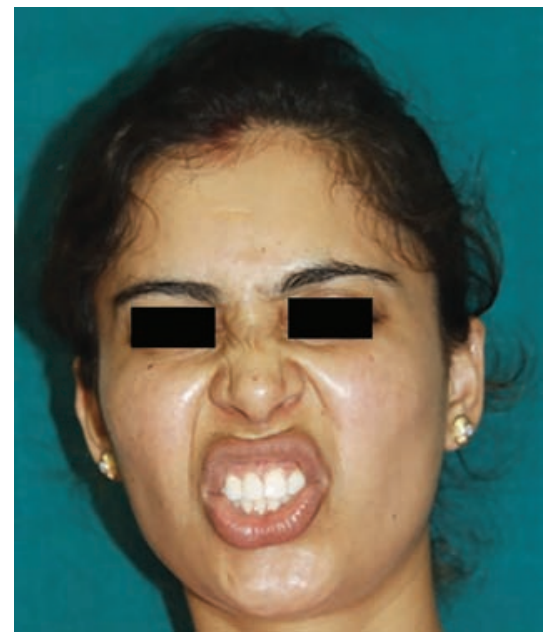

Fig. 4: Example of funnel-shaped expression (AUs 9, 22, and 25)

\section{RESULTS}

Statistically significant differences among the four facial expressions were noted. The median (interquartile range), minimum, maximum, and mean standard deviation values of vertical gingival display of above four maxillary tooth types are shown in Graph 1.

The analysis of variance test followed by post hoc test (Tukey honest significance test) revealed significant mean differences between group A (I), group A(II), group B (I), and group B (II) at 95\% confidence interval (CI), and were considered to be statistically significant.

Paired t-test used to compare the overall pairwise differences in values on right and left sides was considered to be statistically nonsignificant ( $p$-value $\geq 0.05$ ).

One sample t-test showed insignificant differences between golden percentage for both sides at 95\% CI.

Individual t-test showed significant differences between each group at $95 \% \mathrm{CI}$.

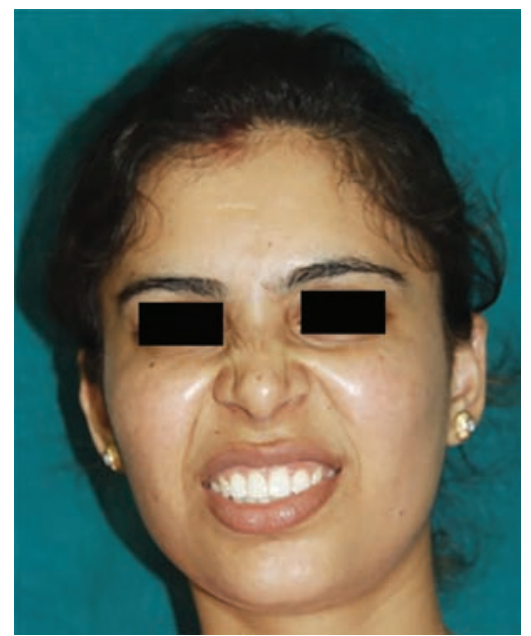

Fig. 3: Example of intense grimace of disgust (AUs 9, 10, and 25)

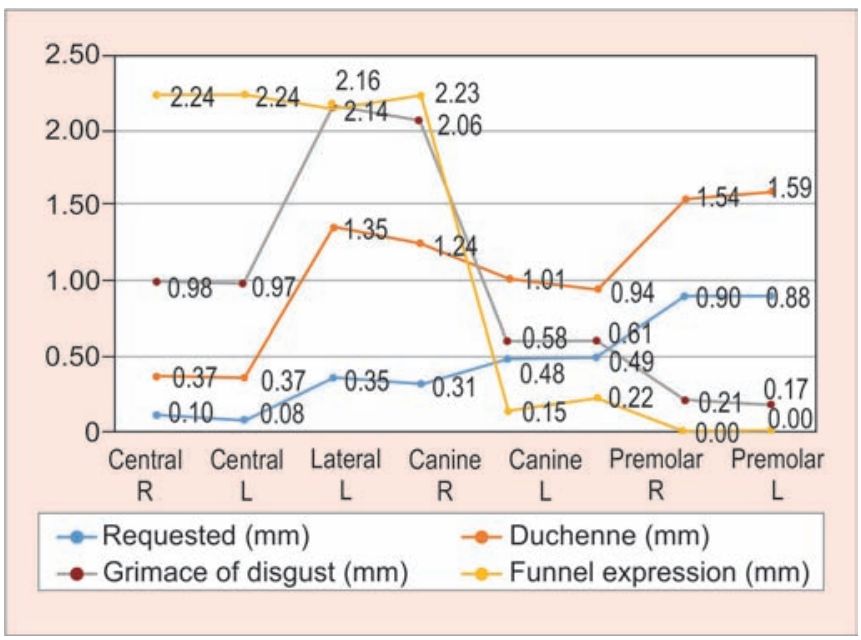

Graph 1: Amount of gingival display over each tooth in four facial expressions

Both the funnel-shaped expression (mean 2.24) and the intense grimace of disgust (mean 0.97, 0.98) revealed significantly more visible gingiva than the Duchenne smile (mean 0.37, 0.35) and the requested "give me your biggest smile" (mean $0.10,0.08$ ) over the maxillary central incisors for both sides.

At the maxillary lateral incisor, the same trend was observed. The funnel-shaped (mean 2.16, 2.23) and the grimace of disgust (mean 2.14, 2.06) showed greater amounts of gingiva than the Duchenne smile (mean $1.35,1.24$ ) and requested smile (mean 0.35, 0.31) for both sides.

In contrast, at the maxillary canines, the Duchenne smile (mean 1.01, 0.94) revealed significantly more gingiva than the intense grimace of disgust (mean 0.58 , 0.61 ) and the funnel-shaped expression (mean $0.15,0.22$ ). In addition, the "give me your biggest smile" (mean $0.48,0.49$ ) displayed significantly more gingiva than the funnel-shaped expression (mean 0.15, 0.22). 


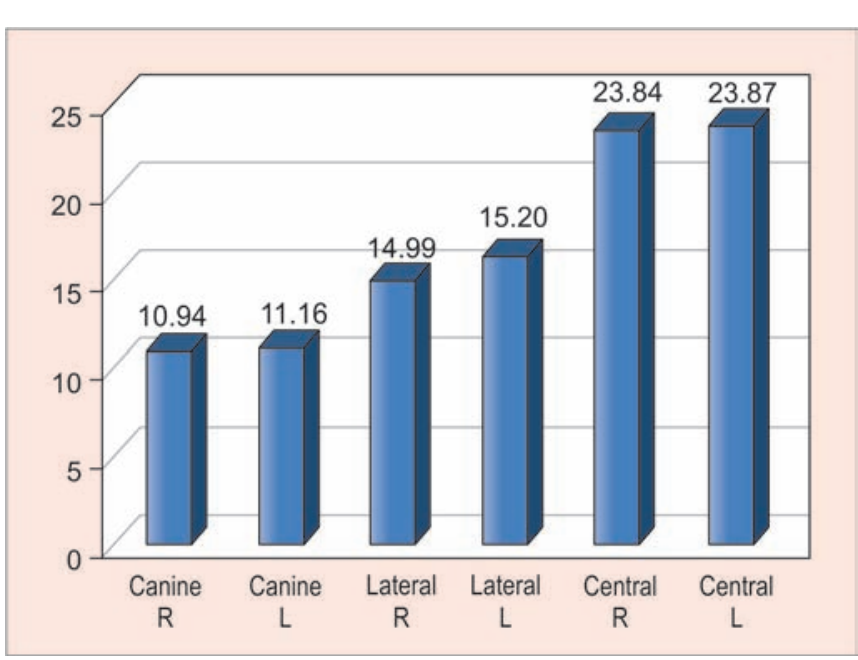

Graph 2: One sample t-test for golden percentage showed no differences between the values obtained and was statistically significant at $95 \% \mathrm{Cl}$

At the maxillary first premolars, it was found that the Duchenne smile (mean 1.54, 1.59) and the requested smile (mean $0.90,0.88$ ) revealed more gingiva than the intense grimace of disgust (mean $0.21,0.17$ ) and the funnel-shaped expression (mean 0.00) (Graphs 1 and 2).

\section{DISCUSSION}

Smile is rightly considered a valuable tool of nonverbal social communication, a civilized form of human contact, and a sound criterion of facial attractiveness. Prosthodontists regard the smile as an integral part of treatment planning and as the cornerstone of treatment objectives. As the mouth is the center of communication in the face, the smile plays an important role in facial expression and appearance. ${ }^{11}$ The gingival margins of the central incisors are normally at the same level or slightly lower than those of the canines, while the gingival margins of the lateral incisors are lower than those of the central incisors. ${ }^{12-14}$ An optimal smile is characterized by an upper lip that reaches the gingival margins, with an upward or straight curvature between the philtrum and commissures; an upper incisal line coincident with the border of the lower lip; minimal or no lateral negative space; a commissural line and occlusal frontal plane parallel to the pupillary line; and harmoniously integrated dental and gingival components. ${ }^{15-17}$ There is always a ratio between large and small called the golden proportion, but it is not always evident and variations are often apparent. ${ }^{18}$ Unfortunately, golden proportion analysis, traditionally, has been applied for unilaterally correlating the width of the canine and central incisor to the width of the lateral incisor on the same side. Unilateral analysis of a smile presents an obvious difficulty: It allows for analysis of dominance and proportion, but not symmetry. ${ }^{5}$ So, golden percentage comes into existence wherein the proportional width of each tooth should be: Canine 10\%, lateral 15\%, central $25 \%$, central $25 \%$, lateral $15 \%$, and canine $10 \%$ of the total distance across the anterior segment, in order to achieve an esthetically pleasing smile. ${ }^{19}$ Kapagiannidis et $\mathrm{al}^{20}$ used still photographs in his study on gingival display in the premolar area during smiling.

Van der Geld et $\mathrm{al}^{21}$ concluded that maxillary lip line heights during spontaneous smiling were significantly higher than during posed smiling. Geron and Atalia ${ }^{22}$ conducted a study on the perception of oral and smile esthetics with different gingival displays and incisal plane inclinations and concluded that the images with exposure of at least $1 \mathrm{~mm}$ of gingiva above the maxillary teeth were found to be less attractive and the amount of upper and lower gingival display was increased during smiling and speech. Al-Jabrah et $\mathrm{al}^{23}$ concluded that only $22 \%$ of the subjects displayed gingiva during a maximum smile. In the age group of 21 to 25 years, only $7 \%$ females and $6 \%$ males displayed gingiva. Subjects who displayed gingiva during a maximum smile had significantly more horizontal and vertical overlap of anterior teeth than compared with subjects who did not show their gingival tissues when smiling. Levin ${ }^{4}$ stated that there exists a golden proportion between a larger part and a smaller part. Lombardi $i^{24}$ proposed that while selecting the size of the teeth, it is necessary to consider the element of proportion, i.e., the golden mean. He suggested that further studies were required to determine the presence or absence of specific width of the tooth ratio, i.e., pleasing. Snow ${ }^{5}$ advocated the use of the "golden percentage" as a means of applying the golden proportion across the midline to encompass the total canine to canine width (six anterior teeth). According to him, the proportional width of each tooth should be: Canine 10\%, lateral 15\%, central $25 \%$, central $25 \%$, lateral $15 \%$, and canine $10 \%$ of the total distance across the anterior segment. Calçada et $\mathrm{al}^{25}$ studied the anterior maxillary teeth proportions of a Portuguese population and concluded that the proportions between the widths of the maxillary anterior teeth were not constant as proposed by the golden proportion and the recurring esthetic dental (RED) proportion. The values observed were closer to the golden percentage. The golden proportion and the RED proportion are unsuitable methods to relate the widths of the maxillary anterior teeth.

The null hypothesis for the study was that no difference would be found in the amount of maxillary gingival display among the four facial expressions evaluated at the four types of teeth. Based on the results obtained, the null hypothesis was rejected. The results of the current study showed that the funnel-shaped expression produced greater gingival display than the other facial expression at the maxillary central and lateral incisors. In the 
present study, it was found that within group A (smile), significant difference was obtained between group AI (requested smile) and AII (Duchenne smile), with values of latter being greater for each tooth on both sides. All values were significant at $99 \% \mathrm{CI}$, thus revealing that both types of smiles should be equally considered to evaluate gingival display over canines and premolars, while Duchenne smile shows greater degree of maximal gingival display as compared with requested smile. Also, within group B (expression), a significant difference was observed between group BI (grimace of disgust) and BII (funnel-shaped expression). All values were significant at 95\% CI, thus revealing both types of expressions should be equally considered to evaluate gingival display over central incisor and lateral incisor. The results of this study showed that the midfacial soft tissues had more vertical movement with a grimace than with a smile. The mean width of gingiva in an average smile was $0.35 \pm 0.37 \mathrm{~mm}$ at maxillary central incisor, $1.35 \pm 1.24$ at the lateral incisor, $1.01 \pm 0.94$ at the canine, and $1.54 \pm 1.59$ at the first premolar. Values obtained from paired t-test between left and right sides for each tooth and for each group revealed an insignificant difference between left and right sides of the face in maximal gingival display. Thus, it shows that the values cannot be compared unless there is gross facial asymmetry or maxilla-mandibular defect involving the oral musculature and lips, in which values obtained would be significantly different for left and right sides of the face.

In the present study, golden percentage values were calculated for group AII (Duchenne smile) because it is the spontaneous smile or the smile, i.e., obtained out of enjoyment. Wider arches displayed lateral incisors and canines that comprised a larger percentage of the perceived canine-to-canine width relative to the central incisors. Therefore, these arches had less dominant central incisors. Arches with symmetric width percentages that deviated from the golden percentage (with central incisor percentages significantly less than $25 \%$ ) had less dominant central incisors and imply a dental arch, i.e., relatively wider.

From the ratio obtained, the percentage was calculated for each tooth on both sides and was compared with the set values using one sample $t$ test. Mean difference obtained was significant at 95\% CI. Thus, obtained values are comparable to the golden percentage for each tooth on each side with a mean difference of central incisor $(\mathrm{R}=-1.1564, \mathrm{~L}=-1.1283)$, lateral incisor $(\mathrm{R}=-0.0128$, $\mathrm{L}=0.1995)$, and canine $(\mathrm{R}=0.9444, \mathrm{~L}=1.155)$.

\section{CONCLUSION}

Within the limitation of the study, it was concluded that there is a significant difference in gingival display between different smiles and different facial expressions, with maximal gingival display best seen over central incisor and lateral incisor while making expressions (grimace of disgust, funnel-shaped expression), whereas it is best seen over canine and premolar while smiling (requested smile, Duchenne smile). This study helps to provide a guideline whereby the prosthodontist can assess the esthetic potential of a complete denture while making new dentures or when confronted by a denture wearer who is unsatisfied with the esthetics of his/her current prosthesis, especially those patients who present with the "denture look" and need design of an esthetic prosthesis. It will help in management of patients with excessive gingival display and will also enable the dental technician to incorporate the necessary esthetics into the prosthesis during design and fabrication. More number of females were evaluated for the present study, as they achieved the facial expressions more accurately than males following the AU system. The golden percentage is seen in almost $99 \%$ of the subjects, thus making it a reliable method with insignificant differences with the fixed values.

There exists no correlation between gingival display between four different facial expressions and golden percentage.

Thus, it can be inferred that there exists a difference in gingival display among four different facial expressions over each tooth visible, and golden percentage was found to be present in more than $99 \%$ of the subjects studied.

\section{REFERENCES}

1. Mack MR. Perspective of facial esthetics in dental treatment planning. J Prosthet Dent 1996 Feb;75(2):169-176.

2. Morley J, Eubank J. Macroesthetic elements of smile design. J Am Dent Assoc 2001 Jan;132(1):39-45.

3. Sarver DM, Ackerman MB. Dynamic smile visualization and quantification: Part 2. Smile analysis and treatment strategies. Am J Orthod Dentofacial Orthop 2003 Aug;124(2):116-127.

4. Levin EI. Dental esthetics and the golden proportion. J Prosthet Dent 1978 Sep;40(3):244-252.

5. Snow SR. Esthetic smile analysis of maxillary anterior tooth width: the golden percentage. J Esthet Dent 1999;11(4):177-184.

6. Ekman P, Davidson RJ, Friesen WV. The Duchenne smile: emotional expression and brain physiology. II. J Pers Soc Psychol 1990 Feb;58(2):342-353.

7. Terry DA, Snow SR, McLaren EA. Contemporary dental photography: selection and application. Compend Contin Educ Dent 2008 Oct;29(8):432-436, 438, 440-442 passim;quiz $450,462$.

8. Ahmad I. Digital dental photography. Part 6: camera settings. Br Dent J 2009 Jul;207(2):63-69.

9. Ahmad I. Digital dental photography. Part 2: purposes and uses. Br Dent J 2009 May;206(9):459-464.

10. Walter RD, Goodacre BJ, Goodacre CJ, Naylor WP, Oyoyo U. A comparison of gingival display with a requested smile, Duchenne smile, grimace of disgust, and funnel-shaped expression. J Prosthet Dent 2014 Aug;112(2):220-227. 
11. Thompson LA, Malmberg J, Goodell NK, Boring RL. The distribution of attention across a talker's face. Discourse Process 2004 Jul;38(1):145-168.

12. Miron $\mathrm{H}$, Calderon S, Allon D. Upper lip changes and gingival exposure on smiling: vertical dimension analysis. Am J Orthod Dentofacial Orthop 2012 Jan;141(1):87-93.

13. Bowers GM. A study of the width of attached gingiva. J Periodontol 1963 May;34(3):201-209.

14. Peck S, Peck L, Kataja M. The gingival smile line. Angle Orthod 1992 Summer;62(2):91-100, discussion 101-102.

15. Sabri R. The eight components of a balanced smile. J Clin Orthod 2005 Mar;39(3):155-167.

16. Brunetto J, Becker MM, Volpato CA. Gender differences in the form of maxillary central incisors analyzed using AutoCAD software. J Prosthet Dent 2011 Aug;106(2):95-101.

17. Loi H, Nakata S, Counts AL. Influence of gingival display on smile aesthetics in Japanese. Eur J Orthod 2010 Dec;32(6):633-637.

18. Hasanreisoglu U, Berksun S, Aras K, Arslan I. An analysis of maxillary anterior teeth: facial and dental proportions. J Prosthet Dent 2005 Dec;94(6):530-538.

19. Murthy BV, Ramani N. Evaluation of natural smile: golden proportion, RED or Golden percentage. J Conserv Dent 2008 Jan;11(1):16-21.
20. Kapagiannidis D, Kontonasaki E, Bikos P, Koidis P. Teeth and gingival display in the premolar area during smiling in relation to gender and age. J Oral Rehabil 2005 Nov;32(11): 830-837.

21. Van Der Geld P, Oosterveld P, Berge SJ, Kuijpers Jagtman AM. Tooth display and lip position during spontaneous and posed smiling in adults. Acta Odontol Scand 2008 Aug;66(4): 207-213.

22. Geron S, Atalia W. Influence of sex on the perception of oral and smile esthetics with different gingival display and incisal plane inclination. Angle Orthod 2005 Sep;75(5): 778-784.

23. Al-Jabrah O, Al-Shammout R, El-Naji W, Al-Ajarmeh M, Al-Quran AH. Gender differences in the amount of gingival display during smiling using two intraoral dental biometric measurements. J Prosthodont 2010 Jun;19(4):286-293.

24. Lombardi RE. The principles of visual perception and their clinical application to denture esthetics. J Prosthet Dent 1973 Apr;29(4):358-382.

25. Calçada D, Correia A, Araújo F. Anthropometric analysis of anterior maxillary teeth with digital photography - a study in a Portuguese sample. Int J Esthet Dent 2014 Autumn;9(3): 370-380. 